\title{
Bionic Inspired Decision Making Unit Model for Autonomous Agents
}

\author{
H. Zeilinger*, T. Deutsch*,Member. IEEE, B. Müller* and R. Lang* Member, IEEE \\ *Vienna University of Technology/Institute of Computer Technology, Vienna, Austria \\ \{zeilinger, deutsch, mueller, langr\}@ict.tuwien.ac.at
}

\begin{abstract}
Modern intelligent control systems for building automation, ambient living or surveillance control have to deal with upcoming requirements like increasing complexity of sensor data, or supporting human beings at their jobs. Therefore the system has to decide on actions taken in case of unforeseen situations. The most complex as well as effective decision making unit that is known, is represented by the human mind. Therefore it seems to be feasible to emulate the human mind in order to design a system for approximating its abilities. While in current Artificial Intelligence a number of generally accepted models, which rely on psychological or neurological theory, are known this paper will introduce a different approach. As a result of the interdisciplinary work of engineers and psychoanalysts a functional model of the human mind has been designed on the base of psychoanalytical theory. As result this work describes the first version of this model in detail and gives an overview on the psychoanalytical theory it relies on.
\end{abstract}

\section{INTRODUCTION}

Current building automation control systems have to cope with a number of upcoming requirements. As a result of rising complexity in sensor networks they are overtaxed by analyzing and evaluating the amount of data they receive [1]. In addition challenging tasks like monitoring in ambient living or surveillance control at crowded places (e. g. airport, convention centers) outrun the opportunities of intelligent control systems. This is due to the requirement of managing unknown situations which poses a big challenge for researchers. Summarized the ideal control system deals with an enormous amount of perceived data, evaluates this data on their relevance, and reaches a decision considering the current environmental situation as well as former experiences. The

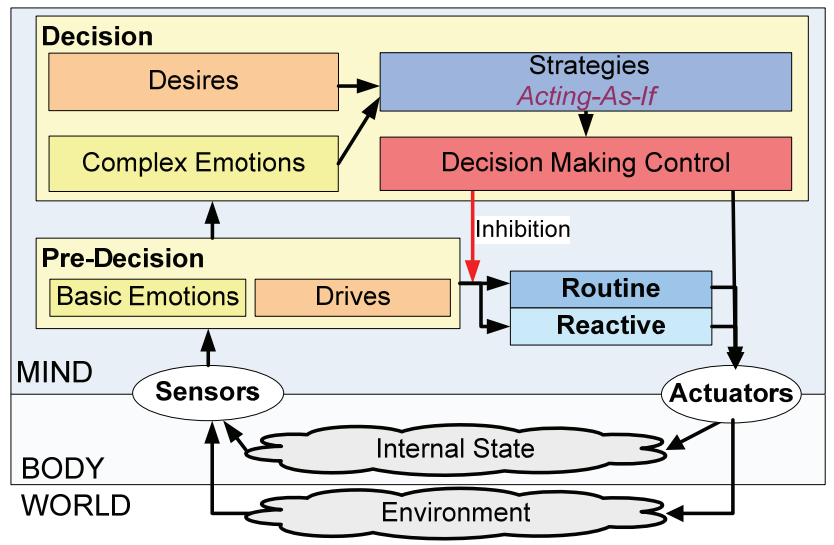

Fig. 1 First ARS-PA Model only system known that fits to these demands is the human mind.

AI (Artificial Intelligence) and CS (Cognitive Science) provide a number of approaches like, neural networks, Bayesian networks, vector machines, or behavior-based models in order to emulate human abilities [2].

Several theories on the human brain's structure and its evolving functions exist in the areas of neurology or psychology even no adequate archetype has been found for implementation yet. The existing models focus on single processes and functions, thus a combination to a technical model leads to inconsistencies. Especially the use of neural networks is limited by the lack of knowledge on the evolvement of higher mental functions out of a combination of numerous neurons. Therefore this work describes a different approach - by the use of a top-down approach the humans' mental functions as they are described in the theories of psychoanalysis are merged to a model without considering the specific structure of the brain's physiology. This model is entitled as new ARS-PA (Artificial Recognition SystemPsychoAnalysis) model.

The project ARS (Artificial Recognition System) [3,4] was introduced in 2003 at the Vienna University of Technology's Institute for Computer Technology in order to take a new line dealing with this issue - using a functional approach on the base of psychoanalytic and neuropsychoanalytic theory. It has been tried to merge neuropsychoanalytic archetypes [5] that were used for system perception and psychoanalytic archetypes that were used as an archetype for decision making, to one model. These efforts resulted in the first ARS-PA model (see Fig. 1). In the course of the research work it became clear that inconsistencies could not be avoided as long as the modeling base is formed by numerous different theoretical models even though these models do not contradict each other it is not for sure that they are consistent. Therefore the new ARS-PA model implements the theory of one single archetype Sigmund Freud's $2^{\text {nd }}$ topographical model [6].

First this work gives a short overview on the first ARS-PA model. While Chapter II presents current state of the art, Chapter III discusses the method of designing the new model in detail. A psychoanalytic explanation of the $2^{\text {nd }}$ topographical model's theory follows in Chapter IV. Chapter V describes the emerged technical model. As a result the conversion of a psychoanalytic model to technical understandable terms has to be seen. The authors understand this article as theoretical work. The result is a framework which has to be filled in future 
research. The importance is within the transformation of psychoanalytical theories into technical feasible terms. The framework will be filled partially by further theories of psychoanalysis. Where this is not possible, models which do not contradict psychoanalysis will be used. An outlook on further work closes this article.

\section{Prerequisites AND StATE OF THE ART}

In 1989 Sherry Turkle described in the article [7] the possibility of a new alliance between artificial intelligence and psychoanalysis. Affective computing, as described in [8] were rarely present before that time and the use of emotions as a basic valuing system for autonomous agents was still in its childhood. The functional way of description as provided by psychoanalysis is a good starting point in developing new, technical approaches to AI. Concepts like Ego-Super-Ego-Id [6] or different views on existing concepts like emotions can be used and have the advantage that they are pre-structured and organized in a way that modern design principals like unified modeling languages in electrical engineering and computer sciences can be used for further technical descriptions. Other concepts within psychoanalysis like sexuality are more difficult to adapt to processes that are commonly described and use in the technical fields except of areas like biological cell farms or the like. Thus, a subset of it called psychodynamics is used [9], that reduces the theories of psychoanalysis by the reproduction aspect and focus especially to the tension, several inner values of an individual, like drives and emotions evoke. This leads commonly to a evaluation system, where a measure of lust is gained from releasing the tension. These models vary commonly in their way on how the tensions are varying and evoked.

In [10] and [11] the first psychoanalytically inspired model shown in Fig. 1 - as developed by the project ARS is described. Concepts like sexuality do not appear in this model, but they are set aside - other than in psychodynamics - for the moment. The model divides the world, an autonomous agent is placed in and therefore can be perceived into the body and the environment. Sensors for the body are able to detect bodily needs like energy. The inner- and outer-world (see Chapter IV) information collected by the sensors is processed in the 'Predecision' unit using emotions and drives as evaluation systems. If needed, the 'Pre-decision' can evoke immediate reactive actions or provide the execution of routine actions with sensory information. The evaluated information is further processed within the decision. Complex or social emotions and desires are leading to more complex behaviors tendencies. In 'ActingAs-If Strategies' these tendencies are put to a test whether they are possible and promising. Further, a set of social rules is searched to prevent behaviors that will lead to disapproval by the other entities within the world (agents, humans, etc.). The module 'Decision' is able to inhibit actions invoked by predecisions. Finally, actuators are executing the selected actions.

The psychoanalytical elements Ego, Super-Ego and Id are hidden within this model. 'Pre-decision' can be seen as the Id, decision as the Ego. The Super-Ego accords to the set of social rules mentioned above.
To be able to test the developed model, a game of artificial life was designed- the so called Bubble Family Game [12]. It is a simple virtual environment consisting out of obstacles and landscapes. AEAs (Autonomous Embodied Agents) roam through it for the search of energy. Some of them provide energy, others consume energy, and some do both at the same time. Each AEA has the first ARS-PA model incorporated within its decision unit. Although, each one of them may be based on the same set of initial rules and values, they evolve differently due to their individual experiences. The simulator allows inspecting the current internal state of each agent. Hence, it supports the development of the model by observing its behavior in well defined situations.

\section{METHODOLOGY}

As mentioned in the introduction, most cognitive models currently existing are inconsistent regarding the different sciences used as archetypes. Behaviorism may not be consistent with findings from psychology or neurosciences'. The robot Kismet is a good example for such a mixture of concepts. It is constructed using design hints from animals, humans, and infants [13]. While the overall achievements of the project are undoubted, the question whether the used theories can be mixed or not has to be raised. To answer it, one has to have profound knowledge in all combined fields of science, which seems to be ridiculous. It is hard to bring scientists of different areas together, whether it is because of complete different views or contradictoriness of their theories. As the main focus of projects like Kismet and ARS lies within constructing a robot and not to start a new scientific field in social sciences, a differentiated approach is needed.

In project ARS, an advisory board of psychoanalysts is responsible for keeping sure that the theories used from sciences other than engineering remain consistent. As psychoanalysis is the main archetype used, the board further monitors that the technical solutions are not in contradiction with it.

The first developed model (see Fig. 1) has been designed obeying this rule. Nevertheless, two main problems arouse. First, many of the introduced non psychoanalytical concepts did not contradict psychoanalysis, but are described using terms which are differently defined in psychoanalysis. For example, the concept of basic emotions as described in [14] has been adapted to our model in [15]. Emotion is a term regularly used by neurologists. Further, the term of feelings is used [16]. In psychoanalysis, these terms are not common. Instead the term affects is used for both of them. Resulting, basic emotions and feelings are not contradicting psychoanalysis, but they get in the way with each other. Second, due to the fact that in psychoanalysis does not exist any clear distinction between flow of control and flow of data, the resulting model lacked of a clear distinction, too.

To overcome these problems, it has been decided to stick within one theory - the $2^{\text {nd }}$ topographical model as defined by Sigmund Freud - and to use a different design approach - TopDown-Design. 
Top-Down is a design approach which begins with the plain idea of starting the modeling of a system at the top level and dividing it into more detailed parts - so called sub modules - in the next lower level. The process of dividing a part into more detailed subparts in the next levels is repeated until the desired grade of detail has been reached. The sum of each level represents the idea as a whole but in different granularities. This design technique fits into Sigmund Freud's description of the $2^{\text {nd }}$ topographical model that unitizes the mind into different modules and sub-modules.

\section{Psychoanalytic Model of the Human Mind}

As the ARS-PA model explored later on refers to Freud's $2^{\text {nd }}$ topographical model it will be described in this chapter from the psychoanalytic point of view.

While in the first topographical model Sigmund Freud made a distinction between three levels of consciousness unconscious, preconscious and conscious - in 1923 - against the background of his theory of the unconscious conflict Freud conceived the $2^{\text {nd }}$ topographical model which is entitled as the structural model too.

Now, Id, Ego and Super-Ego are the three agencies of the psychic personality:

The Id accords to the unconscious as the Ego accords to the conscious. According to Freud's theory, in the beginning the psychic apparatus consists out of an undifferentiated Id before a structured Ego evolves. The Id "[...]contains everything that is present at birth, that is fixed in the constitution - above all, therefore, the instincts, which originate from the somatic organization and which find a first psychical expression here [in the id] in forms unknown to us."[17]. "It is the dark, inaccessible part of our personality; what little we know of it we have learnt from our study of the dream-work and the construction of neurotic symptoms, and most of that is of a negative character and can be described only as a contrast to the ego. We approach the id with analogies: we call it a chaos, a cauldron full of seething excitations...it is filled with energy reaching it from the instincts, but it has no organization, produces no collective will, but only a striving to bring about the satisfaction of instinctual needs subject to the observance of the pleasure-principle." [18]. This concept is an example for explaining mental phenomena in terms of the opposition of antithetical forces. The Id is unorganized, observes the pleasure principle and it conforms to the primary processes which ignore differences and are oblivious of contradictions, space and time. Primary processes are closely related to 'body language' or 'organ language', i.e. “[...] it is situated between the cognitive processes of the consciousness and the physiological processes taking place in the central nervous system. At least by way of description according to Freud, the primary process can be understood as the connecting link between psyche and soma," [19].

On the other side the Ego observes the reality principle and conforms to the secondary processes which are structured and organized executed.

The Ego means a structural and topographical concept referring to the organized parts of the psychic apparatus. In
[17], Freud provides a summary of the Ego: "In consequence of the pre-established connection between sense perception and muscular action, the ego has voluntary movement at its command. It has the task of self-preservation. As regards external events, it performs that task by becoming aware of stimuli, by storing up experiences about them (in the memory), by avoiding excessively strong stimuli (through flight), by dealing with moderate stimuli (through adaptation) and finally by learning to bring about expedient changes in the external world to its own advantage (through activity). As regards internal events, in relation to the id, it performs that task by gaining control over the demands of the instincts, by deciding whether they are to be allowed satisfaction, by postponing that satisfaction to times and circumstances favourable in the external world or by suppressing their excitations entirely. It is guided in its activity by consideration of the tensions produced by stimuli, whether these tensions are present in it or introduced into it. The raising of these tensions is in general felt as un-pleasure and their lowering as pleasure. It is probable, however, that what is felt as pleasure or un-pleasure is not the absolute height of this tension but something in the rhythm of the changes in them. The ego strives after pleasure and seeks to avoid un-pleasure. An increase in un-pleasure that is expected and foreseen is met by a signal of anxiety; the occasion of such an increase, whether it threatens from without or within, is known as a danger. From time to time the ego gives up its connection with the external world and withdraws into the state of sleep, in which it makes far-reaching changes in its organization".

A thing presentation accords to characterising attributes of an object on the base on sensorial data - word-presentations accord to objects described with symbols. The memory, remembrance, and ideational systems consist out of linked thing- and word-presentations. For human beings wordpresentations often accord to linguistic descriptions.

The Super-Ego is the part in the psychic apparatus in which self-observation, self criticism, and other reflective activities develop. Additional it includes unconscious elements and contains parental impacts however it is not an accurate replica of the parental person. Injunctions and inhibitions emanating from it derive from the subject's past and may be in conflict with his present values.

\section{A TECHNICAL MODEL OF THE HUMAN MiND}

As mentioned above the new ARS-PA model bases on Sigmund Freud's $2^{\text {nd }}$ topographical model. Engineers and psychoanalysts analyzed this model with attention to its functional structure. By the methodology use of the TopDown-Design, layers with different levels of granularities were defined. In this chapter an overview on this model is given even the level of granularity has not been adequate yet. Before the technical model of the human mind can be discussed some psychoanalytic definitions and their meaning in technical systems have to be clarified.

1) Drive: a drive is "[...] the psychical representative of the stimuli originating within the organism and reaching the 


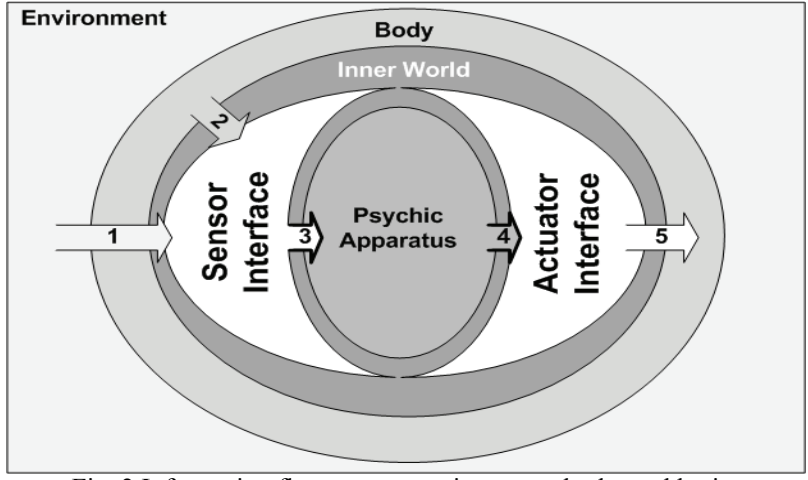

Fig. 2 Information flow among environment. bodv. and brain

mind, as a measure of the demand made upon the mind for work in consequence of its connection with the body" [20].

The drive is responsible for converting physiological stimuli to psychic information. It is activated by a 'bodily need' and initializes a demand which needs to get discharged by the use of an object - this object is called drive-object. The execution of drive-demands can result in conflicts with the current environmental situation or Super-Ego demands. Discharging drive-demands without considering these conflicts may result in endangering the subject. Hence, the 'Ego' is able to inhibit the direct exertion of drive-demands and redirects the tension to other activities. In a technical control system a drive signal will get aroused by a system requirement - e.g. in case of broken hardware or maintenance service.

2) Presentation: As mentioned in Chapter IV, the term 'presentation' defines object characteristics. Two forms of presentations are specified - word-presentations and thingpresentations. While thing-presentations characterize objects with sensorial data, word-presentations accord to characterizations by the use of a symbol set.

3) Affect: In psychoanalysis an affect is defined as the drive-demand's absolute value [20, 21]. The engineering definition of this term accords to a valuation of the drivedemand's relevance.

4) Memory Trace: A memory trace accords to an affect, a thing- or a word-presentation of an object. It serves as pattern for incoming information which activates the trace in case of match.

5) Stimuli: A stimuli accords to a sensorial signal. This signal can be received from the inner- as well as outer-world.

For modeling the new ARS-PA model the partition between outer-world and inner-world has been defined like in the first ARS-PA model. The outer-world accords to the system's environment in combination with the system's body. A system's environment is defined as the area the system has to interact with and where it is used in. Another task is to define a system body. The body is the part of the system that has the ability to interact with the environment even it does not conform to the unit which decides how to interact. The decision making unit is shown in Fig. 2 and accords to the inner world. There all received sensor information is processed, evaluated, and transformed to commands. Hence, the internal world is the subject of discussion in this paper.
The first layer is entitled as 'Inner World' module that is split up into three modules - 'Sensor Interface', 'Psychic Apparatus', and 'Actuator Interface'. Fig. 2 provides a structural overview of the environment and the 'Inner World'. Additional the information flow among the modules is marked by arrows. Environmental and bodily data is received by the system's sensor network - arrows 1 and 2 - and sent to the 'Sensor Interface'. There the data is converted and forwarded arrow 3 - to the 'Psychic Apparatus'.

The 'Psychic Apparatus' represents the technical model of the $2^{\text {nd }}$ topographical model. It processes the incoming sensor data and generates commands to control the actuator functions arrow 4.

The 'Actuator Interface' forwards commands - arrow 5 -, which are generated by the 'Psychic Apparatus', to the executive system units. These commands are executed by the actuator units - represented by the system's body.

Due to the fact that the module 'Psychic Apparatus' contains the technical realization of the $2^{\text {nd }}$ topographical model, its functional description is discussed in detail below. The module 'Psychic Apparatus' is split up in three modules, which are

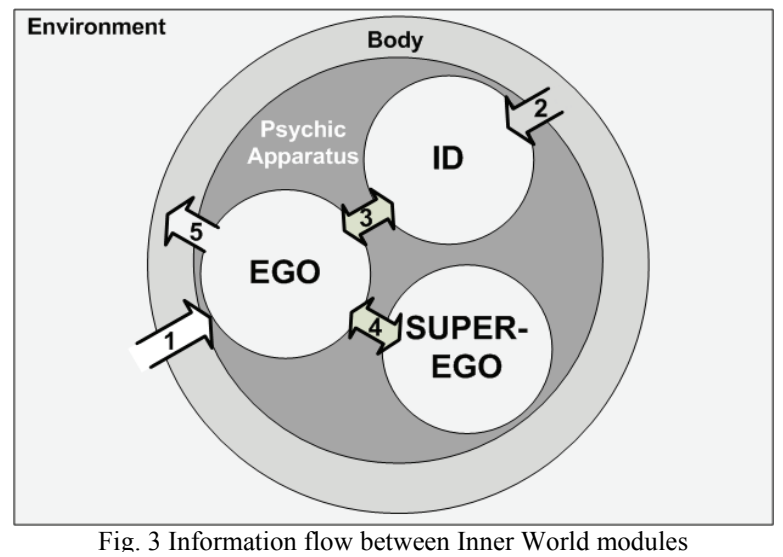

entitled as 'Ego', 'Id', and 'Super-Ego'. Like it is shown in Fig. 3 the 'Ego' and the 'Id' receive information from the outer- world - the 'Id' receives data from the body (see arrow 2) while the 'Ego' receives environmental information (see arrow 1). In addition the 'Ego' is able to send commands to the body (see arrow 5). The duplex inner-world's information exchange is labeled by arrow 3 and arrow 4 .

A. Id

The Id receives information on the system's body. For instance, when an error occurs within the system's specified body, a stimulus arises and a drive is activated. This process converts the 'bodily' stimulus to a message which is interpretable by the 'Psychic Apparatus'. In contrast to a common technical system the 'Id' admits conflictive processes, entitled as primary processes. Whether these processes get handled by the 'Ego' depends on the activation level they are assigned with. The level of activation will be defined by the intensity and importance of drive. For instance, a process, which emerges security or safety issues, will achieve a higher status of priority than a routine procedure. 


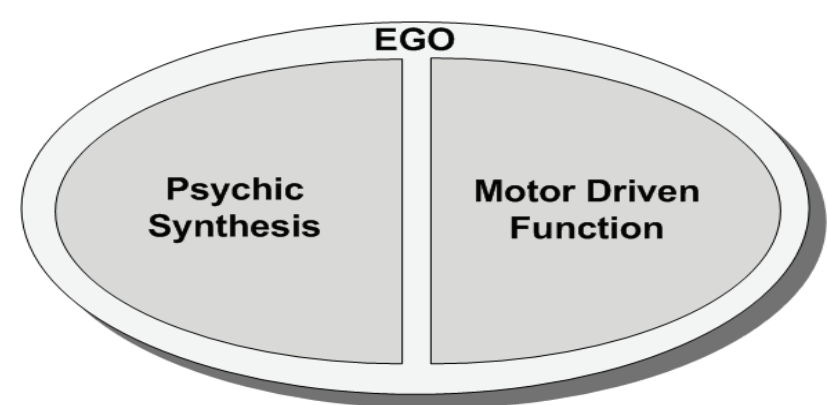

Fig. 4. The functional model of the 'Ego'

The 'Id' is grouped into three functional modules 'Physiological to Psychic Transformation', 'Quantification of Drives', and 'Selection of Memory Traces'.

The 'Physiological to Psychic Transformation' manages the conversion of bodily stimuli to information interpreted by the 'Psychic Apparatus' module.

The 'Quantification of Drives' evaluates the relevance of upcoming drives and passes this information on to the 'Ego' module.

Hence, if an object is perceived at the outer-world, as a result the according memory trace gets activated. This process is executed within the 'Selection of Memory Traces' module. This process is limited to the memory traces which are managed by the 'Id'. The modules 'Ego' and 'Super-Ego' manage memory traces that are used by them on their own.

B. Super-Ego

The 'Super-Ego' represents manages a database, which is formed out of restrictions, demands, and awards. It organizes and implements the description of system tasks, their ideal execution, and safety as well as security rules. The 'SuperEgo' receives an image of the current environmental situation by the 'Ego' and provides an according feedback. As long as the system works within normal parameters the relation between the 'Super-Ego' and the 'Id' will be balanced. If unforeseen events occur the 'Id' will start to deal with upcoming stimuli. If the activity to handle the stimuli disagrees to the 'Super-Ego's' rules a conflict between both modules will emerge. A compromise between both sides has to be found by the Ego module.

The Super-Ego is divided into three modules, which are entitled 'Management of Demands', 'Management of Restrictions', and 'Management of the Ego-Ideal'. Each of these modules selects a preferred action handling within the context of the current environmental situation. This information is retrieved from memory traces. The recommended actions are communicated to the 'Ego'.

The 'Management of Demands' module manages a database of bids, which have to be followed during the execution of tasks. On the contrary the 'Management of Restrictions' module defines restrictions which accord to safety and security issues. Even they are predefined, certain flexibility should be applied to them for the technical implementation. In psychoanalysis the ego-ideal handles the ideal image of oneself. It evolves during childhood due to the identification of a subject to its parental person [21].
Within the new ARS-PA model this module is defined as database of actions for predefined situations. The engineer stores information for the ideal execution of specific scenarios. If unidentified situations occur the memory trace that fits the image of the current situation most, is activated. The associated command is sent to the 'Ego'.

Every module gives feedback to the 'Ego' according to the current environmental situation and its idealistic concept of behavior. The 'Ego' module selects one of them.

C. Ego

The 'Ego' module is the decision making unit of the psychic apparatus. All information detected by the sensor network will pass the 'Ego' in some modality. According to psychoanalytical theory the Ego is responsible for the balance in the mind and mediates between the Id's and the Super-Ego's demands [6].

In a technical system it represents the mediator between outerand inner-world on the one hand and balances the innerworld's modules on the other. A number of functions are defined to fulfill this task like perception, or thinking (see Fig. 5). Like it is shown in Fig. 4 the 'Ego' is divided into two modules - 'Psychic Synthesis' and 'Executive Motor Function'. While the 'Psychic Synthesis' module handles the processing of inner- and outer-world information, the 'Executive Motor Function' module takes an actuating position.

The 'Psychic Synthesis' module is responsible for the 'Ego's' balancing functions which are defined as follows. The 'Perception' module serves as pool for outer- and inner-world information. Any data received is collected and evaluated by modules like 'Focus of Attention'.

Due to an arising drive-demand an according object has to be found in order to get used for drive-discharge. Therefore the perceived data has to get evaluated as to specific searching criteria. While this evaluation is executed by the 'Focus of Attention' module the focus of this searching process is actuated by the 'Focus of Attention Management' module, which is a sub-module of the 'Motor Driven Function' unit (see Fig. 4).

Activated drives, whose relevance is high enough to get processed in the 'Ego', are represented by two components presentations and affects. The 'Affect Management' module collects, evaluates, and forwards affects.

The 'Presentation Management' defines and organizes the associations between thing- and word-presentations. If one memory trace is activated by a presentation their linked

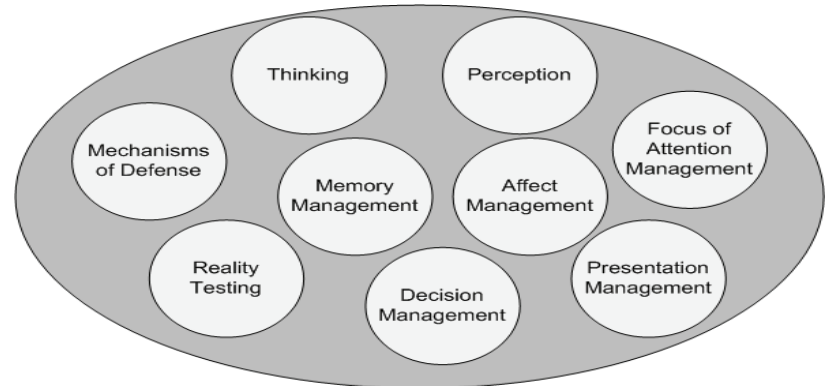

Fig. 5. The functional model of the Psychic Synthesis 
partners are addressed too. Within the 'Ego' only thingpresentations in combination with a word-presentation are processed.

The 'Memory Management' activates, and organizes memory traces which are managed by the 'Ego'. In contrast to the 'Id', beneath thing-presentations, the 'Ego' manages wordpresentations too.

The 'Thinking' module evaluates images of the current outerworld situation and compares them to already defined ones. Existing memory traces, presentations, affects, and facilitations are used as shapes for incoming data. The 'thinking' process structures and organizes the execution of processes and plans of future action handling. Possible action sets are executed within an internal virtual environment and proofed for their plausibility there. Therefore the consequence of executed actions is simulated and evaluated. Reconsidering an action plan is a chance to avoid 'harmful' situations and to manage the system's energy more efficient.

In psychoanalysis the reality test is defined as the function which differentiates between imagination and reality [17]. The same function is assumed for the module 'Reality Testing'. As a consequence to the activation of a memory-trace according to a drive-object, the focus of attention is set to find an equivalent object within the perceived data. If a perceived data activates a similar memory-trace, the 'Reality Testing' module checks the accordance between drive-object and detected object. The obtained data is submitted to the 'Decision Management'.

The 'Decision Management' module has to compare and evaluate the data received from 'Perception' and 'Reality Testing'. It selects the actions that fit the current situation. The resulting commands are sent to the module 'Motility Control', which is placed in 'Motor Driven Function'. They are processed and forwarded to the actuator interface like it is shown in Fig. 2. Demands, which are not considered in the decision, have to be handled another way. As mentioned in [20] every drive-demand has to get discharged. If this discharge cannot be arranged because of occurring conflicts on the one hand, or the environmental situation on the other, the drive-demand has to be redirected by executing substitute acts or temporal inhibiting them. This is done by the 'Mechanisms of Defense' module.

Even though it is for sure that not all of the above described modules will be needed in every technical system it is important to describe one complete model before taking parts of it for implementation.

\section{DISCUSSION AND OUTLOOK}

In this work the new ARS-PA model is discussed theoretically and the basic psychoanalytic theory behind the $2^{\text {nd }}$ topographical model is explained. Additional, the methodology of describing this model in technical useable terms is presented. Finally the new ARS-PA model is discussed, whereas the focus lay on the conversion of psychoanalytical terms to a technical definition - which represents the result of this article. These definitions allocate a concept for ongoing modeling and offer a link for the understanding of psychoanalytical terms in a technical context.
Currently, the research focuses on saving and retrieving memories, decision making, and mechanisms of defense. Even though the new ARS-PA model has to be modeled in a higher level of detail this work shows the idea behind the project and current work. Further work will focus on integrating the model to autonomous agents in order to simulate and analyze its behavior.

\section{REFERENCES}

[1] G. Russ, "Situation-dependent behavior in building automation," $P$ h. D dissertation, Vienna University of Technology, Institute of Computer Technology, 2003.

[2] B. Lorenz and E. Barnard, "A Brief Overview of Artificial Intelligence Focusing on Computational Models of Emotions," Proceedings of the $1^{\text {st }}$ International Engineering \& Neuro-Psychoanalysis Forum, pp. 1-12, 2007.

[3] G. Pratl and P. Palensky, "Project ARS - the next step towards an intelligent environment," Proceedings of the IEE International Workshop on Intelligent Environments, pp. 52-62, 2005.

[4] G. Pratl, D. Dietrich, G. P. Hancke, and W. T. Penzhorn, "A new model for autonomous, networked control systems," IEEE Transactions on Industrial Informatics, vol. 3, pp. 21-32, 2007.

[5] M. Solms and O. Turnbull," The Brain and the Inner World," Karnac/Other Press, Cathy Miller Foreign Rights Agency, London, England, iSBN 1-59051-017-8, 2002.

[6] S. Freud, „Das Ich und das Es,“ Studienausgabe. Psychologie des Unbewußten. Fischer Taschenbuch Verlag GmbH, 1975.

[7] S. Turkle, "Artificial Intelligence and Psychoanalysis: A New Alliance," Graubard, S. R., Ed., Cambridge, MA: MIT Press, pp. 241-268, 1989.

[8] R. W. Picard, “Affective Computing,” The MIT Press, 1997

[9] A. Buller, "Machine psychodynamics: Toward emergent thoughts," Tech. Rep., Kyoto, Japan, 2006.

[10] T. Deutsch, R. Lang, G. Pratl, E. Brainin, and S. Teicher, "Applying Psychoanalytic and Neuro-Scientific Models to Automation," The 2nd IET International Conference on Intelligent Environments, pp. 111-118, 2006.

[11] B. Palensky, "From Neuro-Psychoanalysis to Cognitive and Affective Automation Systems," Ph. D. dissertation, Vienna University of Technology, Institute of Computer Technology, 2008.

[12] T. Deutsch, H. Zeilinger, and R. Lang, "Simulation Results for the ARSPA Model," Proceedings of 2007 IEEE International Conference of Industrial Informatics, pp. 1021-1026, 2007.

[13] C. Breazeal, "Designing Sociable Robots", Cambridge, MA, USA: MIT Press, pp. 42, 2002.

[14] J. Panksepp, "Affective Neuroscience, the Foundations of Human and Animal Emotions," Oxford University Press, Inc. 198 Madison Avenue, New York, 1998, iSBN 0195096738.

[15] W. Burgstaller, R. Lang, P. Poerscht, and R. Velik, "Technical model for basic and complex emotions," Proceedings of 2007 IEEE International Conference of Industrial Informatics, 2007.

[16] A. Damasio, "The Feeling of what Happens. Body and emotion in the Making of Consciousness," Harcourt Brace \& Company, New York, 1999.

[17] S. Freud, "An Outline of Psycho-Analysis," Standard Edition, Hogarth Press, London, 1940.

[18] S. Freud, "New Introductory Lectures on Psycho-Analysis, “ Standard Edition, Hogarth Press, London, 1933.

[19] H. Müller-Pozzi,"Psychoanalytisches Denken. Eine Einführung,“ Verlag Hans Huber, Bern, 2002, pp 66,67

[20] S. Freud, „Trieb und Triebschicksale“ (Instincts and their Vicissitudes), Internationale Zeitschrift für (ärztliche) Psychoanalyse, III, p. 84-100, G.W., X, p. 210-232, S.E. 14: 117-140, 1915.

[21] J. Laplanche, „Das Vokabular der Psychoanalyse,“ Suhrkamp Taschenbuch Wissenschaft, Frankfurt am Main, iSBN 3-518-27607-7, 1973.

[22] P. Schuster and M. Springer-Kremser, „Bausteine der Psychoanalyse,“ WUV Universitätsverlag, 1997. 